\title{
Who like androids more: Japanese or US Americans?
}

\author{
Christoph Bartneck
}

\begin{abstract}
This study investigates to what degree the users' cultural background influences their perception of a robot's anthropomorphism and likeability. More specifically, robots with a conventional robot-like appearance were compared to highly anthropomorphic androids. The US American participants like the robots on average more than the Japanese participants do, but a strong interaction effect was observed between the participants' cultural background and the type of robot. The Japanese participants had a strong preference for conventional robots. This confirms the stereotype that Japanese like conventional robots. However, this does not hold true for highly anthropomorphic androids, which they liked less than the US American participants did. This study focused on the perception of static images of robots and the results may be different for the perception of movies of moving robots or, to an even greater extent, the perception when standing right in front of a moving robot.
\end{abstract}

\section{INTRODUCTION}

$T_{1}$ HE Uncanny Valley theory proposed by Mori in 1970 [1] has been a hot topic in human-robot interaction research, in particular since the development of increasingly human-like androids and computer graphics. In a previous study, we described an empirical study that attempted to plot Mori's hypothesized curve.[2] We focused on highly anthropomorphic androids and even included pictures of real humans, posing as robots. We investigated the influence of framing on the users' perception of the stimuli. Framing had no significant influence on the measurements. The pictures of robots and humans were rated independently of whether the participants knew a particular picture showed a robot or human. The extent to which the stimuli were human-like had a significant influence on the likeability and anthropomorphism measurements, but not even pictures of real humans were rated as being as likeable as the pictures of humanoids or toy robots. As a result, we suggested the existence of an "uncanny cliff" model as an alternative to the uncanny valley model. Two questions remained open: a) to what degree does the cultural background of the participants influence the measurements and b) could the type of robot possibly interact with the cultural background.

Manuscript received January $31^{\text {st }}, 2008$.

Christoph Bartneck is with the Department of Industrial Design, Eindhoven University of Technology, Den Dolech 2, 5600MB Eindhoven, The Netherlands (phone: +31402475175; fax: +31402473285; email: c.bartneck@tue.nl).
We only included Japanese participants in our original study, but another study indicated that the cultural background of participants may have a significant influence on their attitudes towards robots [3, 4].

We therefore conducted a follow-up study that used the same experimental setup as the original study [3], but also included participants from different cultures. The results of the study by Bartneck et al. [3] showed - in contrast to the popular belief that the Japanese love robots - that the Japanese are concerned by the impact that robots might have on society and that they are particularly concerned about the emotional aspects of interacting with robots. A possible explanation could relate to their greater exposure to robots in real life, and particularly through the Japanese media. The Japanese could be more aware of both the robots' abilities and their shortcomings. Participants from the USA were the least negative towards robots, particularly regarding the aspect of interacting with them. A possible reason could be that they are used to technology and at the same time easy-going when it comes to talking to new people. The study by Bartneck et al. [3] focused largely on Sony's Aibo robot, which was the most widely available consumer robot at that time. However, we are now entering a phase in the development of robots in which the first highly anthropomorphic androids are becoming available. Anthropomorphism refers to the attribution of a human form, human characteristics, or human behavior to non-human things such as robots, computers and animals. Hiroshi Ishiguro, for example, developed highly anthropomorphic androids, such as the Geminoid HI-1 robot (see Fig. 1). Some of his androids are, for a short period, indistinguishable from human beings. [5]

It is not obvious if these new androids will be liked differently, in comparison with toy robots or humanoid robots already available. We were particularly interested in including other forms of highly anthropomorphic pictures as well, deriving from computer animation and computer graphics. We also included pictures of real humans as a way of showing perfectly anthropomorphic entities.

To be able to investigate this question, measurement instruments for the anthropomorphism and likeability of robots are necessary. First, we conducted a literature review to identify relevant measurement instruments. It has been reported that the way people form positive impressions of others is to some degree dependent on the visual and vocal behavior of the targets [6] and that positive first impressions (e.g. likeability) of a person often lead to more 
positive evaluations of that person.[7] Interviewers state that they know within the first 1 to 2 minutes whether a potential job applicant will be hired, and people report knowing within the first 30 seconds how likely it is that a blind date will be a success.[8] There is a growing body of research indicating that people often make important judgments within seconds of meeting a person, sometimes remaining quite unaware of both the obvious and subtle cues that may be influencing their judgments. Therefore it is very likely that humans are also able to make judgments of robots based on their first impressions.

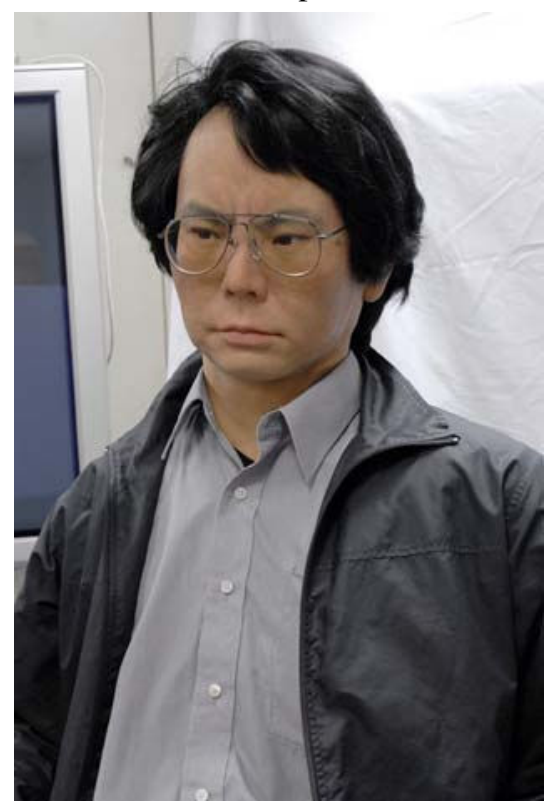

Fig. 1. Geminoid HI-1 robot

Jennifer Monathan [9] complemented her 'liking' question with 5-point semantic differential scales (nice/awful, friendly/unfriendly, kind/unkind, and pleasant/unpleasant) because these judgments tend to be strongly correlated with liking judgments.[10] She reported a Cronbach's alpha of 0.68 , which gives us sufficient confidence to apply her scale in our study.

As well as likeability, we also required a measurement tool for anthropomorphism. Powers and Kiesler [11] used six items and were able to report a Cronbach's alpha of 0.85 . Their questionnaire therefore appears to be more suitable. It was necessary to transform the items used by Powers and Kiesler into semantic differentiators: fake /natural, machine-like/human-like, unconscious/conscious, artificial/lifelike, and moving rigidly/moving elegantly. Two more studies are available in which this new anthropomorphism questionnaire was used. The first one reports a Cronbach's alpha of 0.878.[2] The second reported Cronbach's alphas beween 0.856-0.929.[12] The alpha values are well above 0.7 , so we can conclude that the anthropomorphism questionnaire has sufficient internal consistency reliability.

\section{METHOD}

We conducted a 6 (robot type) x 2 (nationality) x 2 (gender) split-level experiment, with nationality and gender varying at the participant level and each participant being given all robot types. The robot type variable had six values: real human (rh), manipulated human (fh), computer graphic (cg), android (an), humanoid (hd), and toy robot (tr). The previous study included four kinds of highly realistic androids, computer graphics, and humans to investigate the uncanny valley. For this study, it would have been sufficient to focus on only the android, humanoids and toy robots, but since the data of the first study was already available, nothing would be gained by excluding this data. The nationality variable had two values: USA and Japan.

\section{A. Measurements}

We measured the likeability of the stimuli using Monathan's [9] liking question in addition to four of her semantic differential scales: nice/awful, friendly/unfriendly, kind/unkind, and pleasant/unpleasant. However, we deviated from her questionnaire by using a 7-point scale instead of a 5-point scale. To ensure consistency, we converted the human-likeness items found in Powers and Kiesler [11] to 7-point semantic differential scales: fake/natural, machinelike/human-like, unconscious/ conscious, artificial/lifelike. Both questionnaires were translated from English to Japanese. The translation included several cycles of back-translation and revision.

\section{B. Stimuli}

MacDorman [13] presented movie sequences of existing robots to his participants. However, as these robots were shown in different contexts and behaved differently (only some were able to talk), MacDorman concluded that these differences create considerable noise in the measurements. To eradicate this, we picked pictures that focused on the face and did not provide any contextual information.

Another method for creating stimuli for this type of study has been proposed by MacDorman [13] and Hanson.[14] They used sequences of morphed pictures and thereby generated entities that would be impossible to create in reality. We only used pictures of entities that either already exist or that are extremely similar to existing entities, such as computer-generated faces. It can be argued that computer-generated faces are also impossible to observe in reality. However, the artists who generated these pictures focused explicitly on the creation of realistic faces, which is a great challenge in computer graphics.

Hanson pointed out that the beauty of a face already influences its likeability and that therefore great care should be taken to create beautiful androids. To avoid a possible bias we selected pictures of reasonably beautiful women. It was necessary to focus on women because only female androids were available at the start of the study. The first male highly human-like android, Geminoid HI-1 (see Fig. 
1), only became available shortly after the start of this study. Presenting only female androids introduces a gender bias. Female entities might in principle be preferred over male entities. To be able to at least control this bias systematically, we only presented pictures of female entities, at the cost of not being able to generalize to male entities.

To prevent further possible bias by accidentally selecting an extraordinarily beautiful or ugly picture of an android or human, we presented pictures of three different entities from each category, resulting in a total of 18 pictures. The pictures in the android category were of Actroid (Kokoro), EveR 1 (KITECH) and Repliee Q1 (Osaka University). The computer graphics pictures were of Kaya (Alceu Baptistao), Maxim Cyberbabe (Liam Kemp) and an unnamed entity by Young Jong Cho. The manipulated human and real human images were taken from the Elle fashion magazine. The names of the models are unknown. The skin color of the faces in the manipulated human category was adjusted to give it a slightly green hue, producing a mildly artificial look, similar to "Data" (an android character from the television series "Star Trek"). The pictures in the humanoid category were of Qrio (Sony), Asimo (Honda) and an unnamed humanoid from Toyota. The robot pet pictures were of Aibo (Sony), PaPeRo (NEC) and iCat (Philips Research). We are not able to present the pictures used in the experiment in this paper since some companies, such as Sony, refused to give us permission.

\section{Procedure}

The questionnaire was implemented on the internet. Participants could access it using a web browser. After filling out several demographic questions, they had to rate each stimulus, one at a time (see Fig. 2). The participants were told that the pictures shown were robots.

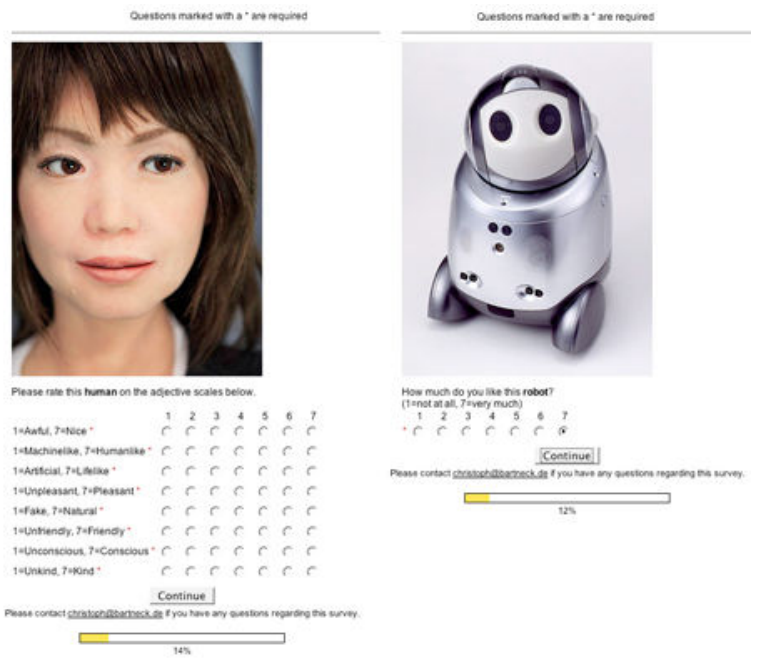

Fig. 2. Two screenshots of the questionnaire.

Each of the 18 stimuli was presented twice: once with the liking question and once with the semantic differential scales. This resulted in the presentation of a total of 36 questions. The order of the 36 questions was randomized.

\section{Participants}

112 participants aged between 18 and 52 years (mean 24.7) filled in the survey. 57 participants were female and 55 were male, while 54 participants had US nationality and 58 held the Japanese nationality. All participants were associated with a University in the Kyoto district of Japan or with the Missouri State University.

\section{RESUlts}

A reliability analysis across all factor values was conducted. The resulting Cronbach's alpha for the anthropomorphism (0.9) and the likeability (0.841) give us sufficient confidence in the reliability of the questionnaires. Furthermore, Levene's test for equality of variance was not significant for any of the measurements; so homogeneous variance can be assumed. Table 1 shows the distribution of the participants across the between-participant factors.

TABLE I

UNITS FOR MAGNETIC PROPERTIES

\begin{tabular}{ccc}
\hline NATIONALITY & MALE & FEMALE \\
\hline USA & 27 & 27 \\
Japan & 28 & 30 \\
\hline \hline
\end{tabular}

The mean over all three images for each of the six robot types was used for the further analysis. We conducted a multilevel analysis of variance (ANOVA) in which the robot type was the within-participant factor. Nationality and gender were the between-participant factors. Figure 3 shows the overall means of anthropomorphism and likeability across all conditions.

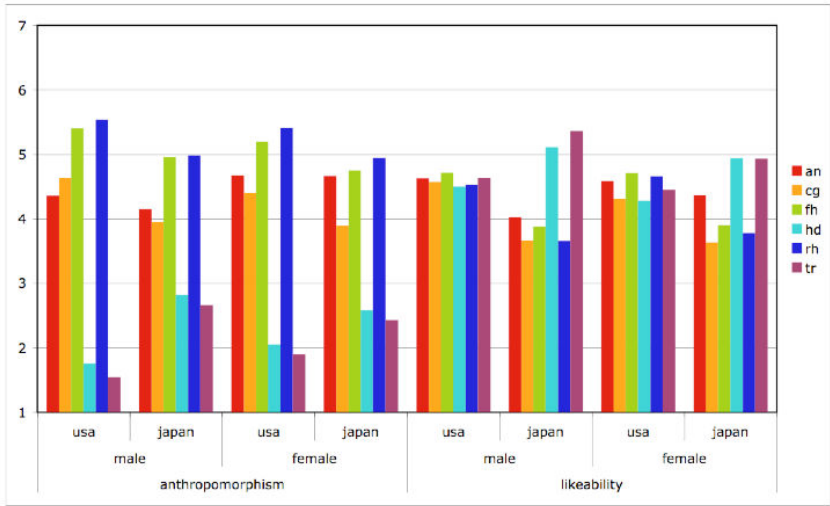

Fig. 3. Mean anthropomorphism and likeability across all conditions.

Gender has no overall significant effect on anthropomorphism $\quad(\mathrm{F}(1,108)=0.1, \quad \mathrm{p}=0.922) \quad$ or $\quad$ on likeability $(\mathrm{F}(1,108)=0.336, \mathrm{p}=0.564)$. There is an almost significant interaction effect between robot type and gender on anthropomorphism $(\mathrm{F}(5,540)=2.089, \mathrm{p}=0.065)$ but not 
on likeability. Male participants had a slight tendency to rate the face as being more human-like than female participants. However, for the android faces, this trend was reversed. The female participants rated the android faces as being more anthropomorphic than the male participants (see Figure 4).



Fig. 4. Mean anthropomorphism across the robot type conditions for male and female.

Nationality has no overall significant effect on anthropomorphism $(\mathrm{F}(1,108)=0.005, \mathrm{p}=0.945)$ but it does have a significant effect on likeability $(\mathrm{F}(1,108)=6.555$, $\mathrm{p}=0.012$ ). The US participants (mean 4.551) liked the faces more than the Japanese participants (mean 4.273).

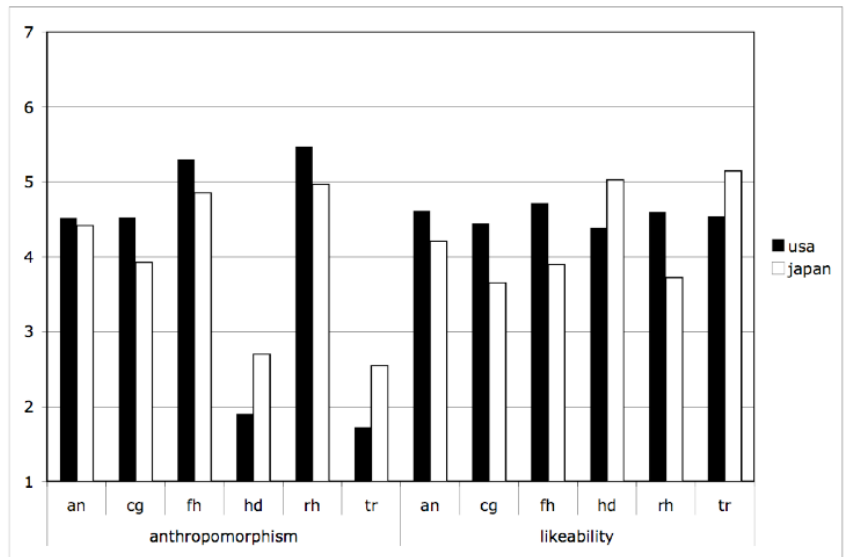

Fig. 5. Mean anthropomorphism and likeability across the robot type and nationality conditions.

There is a highly significant interaction effect between robot type and nationality on anthropomorphism $(\mathrm{F}(5,540)=18.341, \quad \mathrm{p}<0.001) \quad$ and likeability $(\mathrm{F}(5,540)=25.202, \mathrm{p}<0.001)$. The US participants rated the faces higher on anthropomorphism and likeability except for the humanoid and toy robot faces, where the Japanese participants gave the higher ratings for anthropomorphism and likeability (see Figure 5 and 6).

Robot type has significant influence on anthropomorphism $(\mathrm{F}(5,540)=315.211, \mathrm{p}<0.001)$ and on likeability $(\mathrm{F}(5,540)=19.218, \mathrm{p}<0.001)$. Post hoc t-tests with Bonferroni-corrected alpha showed that only three pairs of faces were not significantly different from each other in terms of anthropomorphism : an from cg $(\mathrm{p}=0.162)$, fh from $\mathrm{rh}(\mathrm{p}=0.788$, and hd from $\operatorname{tr}(\mathrm{p}=0.074)$. The same comparison for likeability revealed five pairs that were not significantly different from each other: an with fh $(\mathrm{p}=1.000)$, an with hd $(\mathrm{p}=0.184)$, rh with $\mathrm{cg}(\mathrm{p}=1.000)$, rh with fh $(\mathrm{p}=0.955)$, and hd with $\operatorname{tr}(\mathrm{p}=1.000)$. It appears as if the participants formed mental groups of tr+hd and $f h+r h$. This result is supported by plotting the means for each robot type onto the two-dimensional space of anthropomorphism and likeability (see Figure 6).

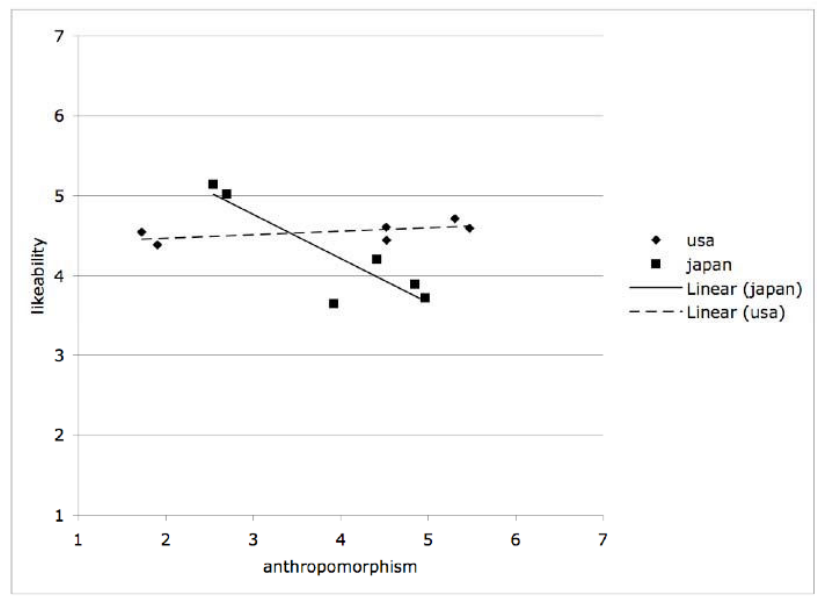

Fig. 6. Means of all robot types plotted onto the anthropomorphism/likeability space.

\section{CONCLUSIONS AND DISCUSSION}

The cultural background, in interaction with the type of robot, had a strong influence on the anthropomorphism and likeability measurements. While the US participants mildly increased their likeability ratings for increasingly anthropomorphic robots, the Japanese participants showed a reverse trend. The more human-like the robots were the less they were liked. For both Japanese and US participants, there is a marked distinction between the clearly robot-like types (tr, hd) and the highly anthropomorphic androids (an, $\mathrm{cg}, \mathrm{fh}, \mathrm{rh})$. This result confirms the stereotype that Japanese like conventional robots. It is possible to exclude the idea that the more negative ratings for the highly anthropomorphic robots are based on racial preferences. Own-race faces are only more attractive than other-race faces for male faces, and the most attractive faces have mixed-race characteristics.[15]

The results of this study open a new perspective on our previous study.[2] The "uncanny cliff" (see Figure 7) we observed might have been limited to the Japanese culture. The strong preference for robots with a robot-like appearance might have formed the cliff. This may be explained by the strong presence of robots in Japanese popular culture, starting with Astro Boy.[16] However, the results of this study confirm our previous result [3] that, in general, US Americans have a more positive attitude towards robots. 


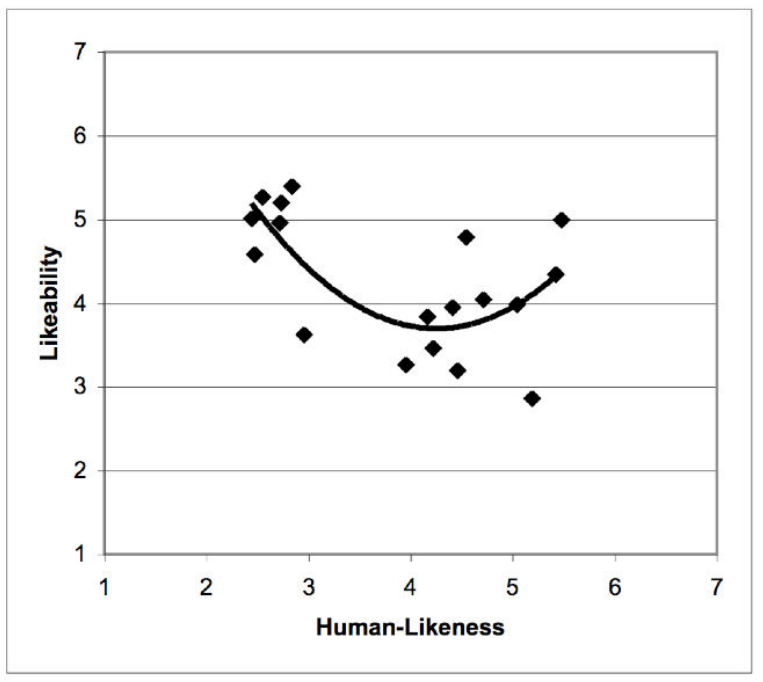

Fig. 7. Means of all pictures plotted onto the humanlikeness/likeability space. (taken from [2])

Overall we can conclude that the cultural background of the users does play an important role in the interaction between users and the robots. The differences between Japanese and US American participants were greater for toy robots and humanoids than for the highly anthropomorphic androids. To answer the question posed in the title of our paper: no, Japanese do not like androids significantly more than US Americans do. But this may change in the future. The Japanese media are filled with stories and pictures about current toy robots and humanoids. Once media coverage increases and people are being exposed to androids, they become familiar with them and the robot-associated eeriness may be eliminated.[17] Given that the likeability of robots changes through the users' exposure to them, we have to acknowledge that this study is only a snapshot. If this study is repeated ten years from now, it is likely to produce different results. But keeping a historical record on how the perception of robots changes over time is still an interesting question and we hope to be able to report on it in the future.

It would also be interesting to compare the results of this study (using static pictures) with those of a similar study using moving pictures. In particular, the comparison between moving androids and humanoids would be of interest. It would be necessary to film various robots and humans attempting to execute the same movements. The camera position, angles and background would all need to be similar. It would be even better to test the users' perception with real robots instead of movies and pictures. The robots' social presence might have a strong effect. After all, the goal of robotics is to bring real robots into our society, not movies and pictures of them.

\section{ACKNOWLEDGMENTS}

I would like to thank Takayuki Kanda and Sheryl Brahnam for supporting this study.

\section{REFERENCES}

[1] M. Mori, "The Uncanny Valley," Energy, vol. 7, pp. 33-35, 1970.

[2] C. Bartneck, T. Kanda, H. Ishiguro, and N. Hagita, "Is the Uncanny Valley an Uncanny Cliff?," in 16th IEEE International Symposium on Robot and Human Interactive Communication, RO-MAN 2007, Jeju, Korea, 2007, pp. 368-373.

[3] C. Bartneck, T. Suzuki, T. Kanda, and T. Nomura, "The Influence of People's Culture and Prior Experiences with Aibo on their Attitude Towards Robots," AI \& Society - The Journal of Human-Centred Systems, vol. 21, pp. 217-230, 2007.

[4] J. J. Wagner, D. M. Cannon, and H. F. M. Van der Loos, "Crosscultural considerations in establishing roboethics for neuro-robot applications," in 9th International Conference on Rehabilitation Robotics. ICORR 2005, 2005, pp. 1-6.

[5] H. Ishiguro, "Android Science - Towards a new crossinterdisciplinary framework," in CogSci Workshop Towards social Mechanisms of android science, Stresa, 2005, pp. 1-6.

[6] N. Clark and D. Rutter, "Social categorization, visual cues and social judgments," European Journal of Social Psychology, vol. 15, pp. 105-119, 1985.

[7] T. Robbins and A. DeNisi, "A closer look at interpersonal affect as a distinct influence on cognitive processing in performance evaluations," Journal of Applied Psychology, vol. 79, pp. 341-353, 1994.

[8] J. H. Berg and K. Piner, "Social relationships and the lack of social relationship," in Personal relationships and social support, W. Duck and R. C. Silver, Eds. London: Sage, 1990, pp. 104-221.

[9] J. L. Monathan, "I Don't Know It But I Like You - The Influence of Non-conscious Affect on Person Perception," Human Communication Research, vol. 24, pp. 480-500, 1998.

[10] J. K. Burgoon and J. L. Hale, "Validation and measurement of the fundamental themes for relational communication," Communication Monographs, vol. 54, pp. 19-41, 1987.

[11] A. Powers and S. Kiesler, "The advisor robot: tracing people's mental model from a robot's physical attributes," in Proceeding of the 1st ACM SIGCHI/SIGART conference on Human-robot interaction, Salt Lake City, Utah, USA, 2006.

[12] C. Bartneck, T. Kanda, H. Ishiguro, and N. Hagita, "My Robotic Doppelgänger - A Critical Look at the Uncanny Valley Theory," Autonomous Robots, 2008.

[13] K. F. MacDorman, "Subjective ratings of robot video clips for human likeness, familiarity, and eeriness: An exploration of the uncanny valley," in ICCS/CogSci-2006 Long Symposium: Toward Social Mechanisms of Android Science, Vancouver, 2006.

[14] D. Hanson, "Exploring the Aesthetic Range for Humanoid Robots," in CogSci Workshop Towards social Mechanisms of android science, Stresa, 2006.

[15] G. Rhodes, K. Lee, R. Palermo, M. Weiss, S. Yoshikawa, P. Clissa, T. Williams, M. Peters, C. Winkler, and L. Jeffery, "Attractiveness of own-race, other-race, and mixed-race faces," Perception, vol. 34, pp. 319-340, 2005.

[16] O. Tezuka, "Astro Boy: The Birth of Astro Boy," N. Ishiguro and O. Tezuka, Eds.: MANGA VIDEO, 2002.

[17] M. P. Blow, K. Dautenhahn, A. Appleby, C. Nehaniv, and D. Lee, "Perception of Robot Smiles and Dimensions for Human-Robot Interaction Design," in 15th IEEE International Symposium on Robot and Human Interactive Communication (RO-MANO6), Hatfield, 2006. 